\title{
Theoretical Design and Development of Novel English Online Education and Learning System based on B/S Architecture
}

\author{
LI Xiaoqin ${ }^{1, \text { a }}$ \\ ${ }^{1}$ Hubei University of Technology, Wuhan 430068, China \\ a Lisy1996@126.com
}

Keywords: English Online Education; B/S Architecture; Structure Modification and Optimization.

\begin{abstract}
In this paper, we design and development of novel English online education and learning system based on B/S architecture. Online learning system mainly relies on the network advantage which will build flexible and convenient communication platform between teachers and students in order to overcome the traditional two-way communication between teachers and students in time and space. Machine learning and storage technique will help to enhance the performance and outcome of the system. We optimize the current data management situation and modify the storage pattern to enhance the performance. The result shows the effectiveness and robustness of the system.
\end{abstract}

\section{Introduction}

At present, teachers and students can use all sorts of internet-based tools as their course teaching and learning methodology. These tools have become very mature, many formal education course from the initial face-to-face instead of part of the online or even completely online. Now most of the teachers and students are equipped with high performance computer and connected to the network so the network education continues to expand rapidly. Online learning system mainly relies on the network advantage which will build flexible and convenient communication platform between teachers and students in order to overcome the traditional two-way communication between teachers and students in time and space, human aspects such as inconvenience. The traditional construction of online education platform is shown as the follows. (1) Teaching content distribution. Teaching content is the main content of online learning, form is not limited to text, sometimes present in the form of multimedia, but under normal circumstances, the teaching content is static, so according to the type and content classification can be published on the website. In addition, the education content systems on the query function. (2) Work function. For homework, students need to pay by means of upload. Teachers should according to the job completion situation for each work. (3) Announcement function. Teachers can function through announcement issued the notice, announcement content can time change and update regularly. (4) Answering function. Non real-time answer: the function of the system need to complete a discussion group so that students to the teacher to ask and answer smoothly; Real-time answer which is extremely similar to a chat room, students and teachers could log on to the system at the same time and the real-time communication [1-2].

The most challenging issues on developing online education platform could be summarized as the following. (1) The database storage optimization. As far as the real-time online education, the data amount waiting for process is large. It is necessary to find out for optimization methodologies to enhance the database performance. (2) The user interface design. (3) The data structure modification and code bug detection. To build up a robust and well-performed system, the mechanism of debugging is more than necessary. Online learning floating system is steamed to technology disc a learning system, teachers' homework through the network, round answer students' questions, announcement, etc. Information and networking, virtualization platform to improve the students' learning efficiency of online learning which will promote students innovations on their ways of learning and develop their ability of independent learning provides a good hardware environment. Online learning system based on ASP using B/S mode has a good reliability and expansibility, the system platform to run on the Internet and broke through the traditional classroom on time and empty 
asked restrictions on learning. Online learning system based on asp, can online learning, teachers' answering questions, online discussion, online assessment can be comparable to those of the traditional teaching functions such as comprehensive module for learners. We optimize the current data management situation and modify the storage pattern to enhance the performance. The system's organization is described as the follows. User systems use various teaching content published by system through teacher module made by learners learning request and subject to the system to provide learning helper for the teaching process to view the acquisition of learning process, based on the system to collect the student's learning process, test scores, learning mentality corresponding guidance to individual learning [3-4]. System of management through the management module of the system management, maintenance, resource updates, revisions, etc. In the following sub-sections, we will discuss the issues in detail with numerical analysis.

\section{The Principles of Developing the System}

The system structure of $\mathbf{B} / \mathrm{S}$. B/S (Browser/Server) mode is a kind of three layer or multilayer structure of distributed system, is by the Browser (Browser) and Server (Server). The Server includes a Web Server, database Server, application Server, such as its structure as shown in figure 1. In this mode, at the request of the client to the Web Server via a Browser by the Web Server to the database Server query request, a Web Server to query data in the form of a hypertext document to the Browser. $\mathrm{B} / \mathrm{S}$ mode application system is a kind of thin client, the client using a single Browser software, hardware configuration requirements is not high; Easy to manage and maintain the system, software development, upgrade and maintenance on the server side only, reduced the development and maintenance work; The system does not need to develop the client software, web browser software can free download from the Internet, free upgrade; The system protection enterprise investment, B/S mode using standard TCP/IP, HTTP protocol, can be combined with the enterprise existing network well. At the same time it has good expansibility, can directly connect the Interne. So B/s mode with its easy to use, easy to maintain, high degree of information sharing are gradually replacing C/S mode [5].

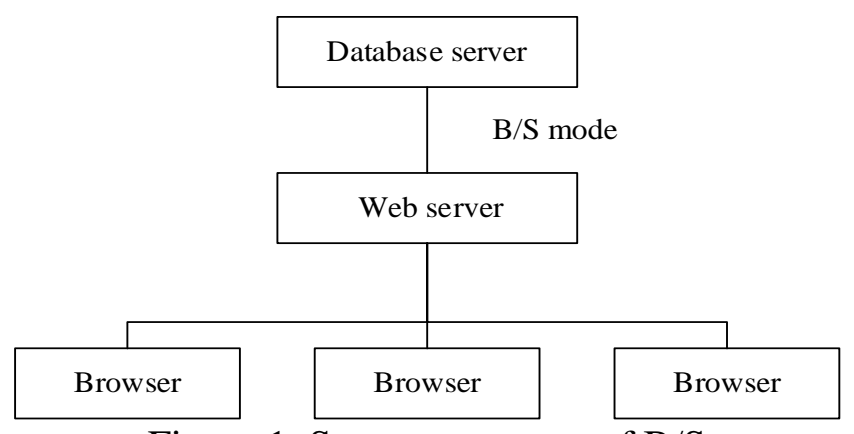

Figure 1. System structure of B/S

Advantage of $\mathrm{B} / \mathrm{S}$ is, first of all, it simplifies the client. It need not like $\mathrm{C} / \mathrm{S}$ mode in different client installed on different client application. Second, it simplifies the development and maintenance of the system. System developers need not again for different levels of user application design and development of different customers, just put all the function of the realization on a Web server, and the different function set permissions for each group of users. Individual users via HTTP request within the scope of authority call different handler on the Web server, so as to complete the data query or modify. Again, it allows the user operation easier.

The Database Optimization Algorithm. Such as artificial intelligence, information technology and pedagogy theory and technology development, design arrangement beforehand good learning process and content of online learning platform have been unable to meet the personalized needs. With so many network at the universities of the vigorous development of open and distance education. Online learning will have more and more users. The advent of the 3G era, mobile devices and the development of wireless communication technology which make people anytime and anywhere online learning becomes more effective and convenient. With huge amounts of data and reduce the 
generation of vast users. The recommended speed system performance will gradually appear. To deal with the severe issue, we adopt the machine learning algorithms shown in the formulas.

$$
\text { Similarity }(S, C)=\left(\sum_{i=1}^{K} W_{i, s} W_{i, c}\right) /\left(\sqrt{W_{i, s}^{2}} \times \sqrt{W_{i, \mathrm{c}}{ }^{2}}\right)
$$

Personalized recommendation technology recommended strategy include: basic recommendations based on knowledge, collaborative filtering recommendation, based on the recommendations from the network structure, based on the recommendations from the content, combined recommendations and other recommendation. It absorbs the object-oriented software engineering for many years in the field of excellent research results, using the new visual modeling. In this paper, the recommendation technology based on content, by comparing the learner interest model and the system of curriculum resources of the library similarity to the learner is recommended which is shown in the following.

$$
\begin{aligned}
& \text { Value }(S)=\text { Fitness }(S)=\operatorname{Similarity}(S, C) \\
& S=\left\{\left(T_{1}, W_{1+w}\right),\left(T_{2}, W_{2+w}\right),\left(T_{3}, W_{3+w}\right), \ldots,\left(T_{n}, W_{n+w}\right)\right\}
\end{aligned}
$$

Before the implementation of genetic algorithms, by extracting learners to read the text features of learning resources from the learner's individual registration information to extract the keywords, the text characteristic and keywords says the current interest in learning, learners concrete method is obtained through data mining technology. Then the text characteristics and keyword synonym for processing, constructing learners' initial interest model according to the personal data form the learner interest figure shown in the figure 2.

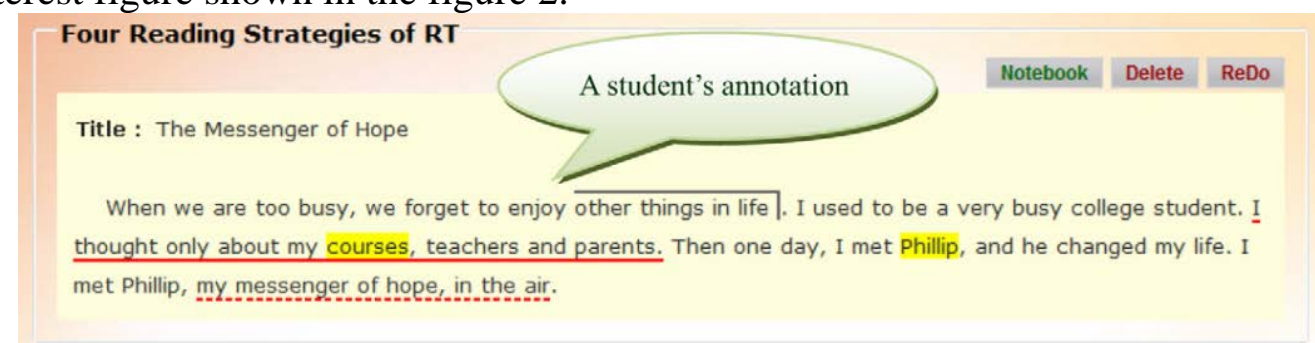

Fig. 2 The Optimization based English Education System

First we get and implement the key use cases the basic framework of the system is established. Again with this frame as the center and in iterative development each iteration only develop use cases, reduced complexity, reduce the cost. The following expressions show this.

$$
\text { representation }(x, i)=\text { occurrences }(x, i)-1
$$

Our paper is a starting point for studying the quality of interface design and the impact of interface design defects on the quality of software application. Our findings implicate researchers and engineers should distinguish classes and interfaces when estimating the quality of software applications. Software engineers must do not create needless sub-typing relations to interfaces. Furthermore, they have to consider the reusability of interfaces and to be more careful while organizing service declarations into interfaces.

The System Development and Implementation Steps. According to the basic ideas of constructivism learning and teaching theory, emphasizes the learners is the center of the learning activities, emphasizes the learners are actively constructing knowledge. Therefore, set the starting point of network teaching design should be positioned in the learner characteristic analysis including the subject for learners to learn about the impact of psychological, physiological characteristics and social understanding and learning style for learners to take especially know learners beginning ability. In the figure 3, we show the general organization of the proposed system. 


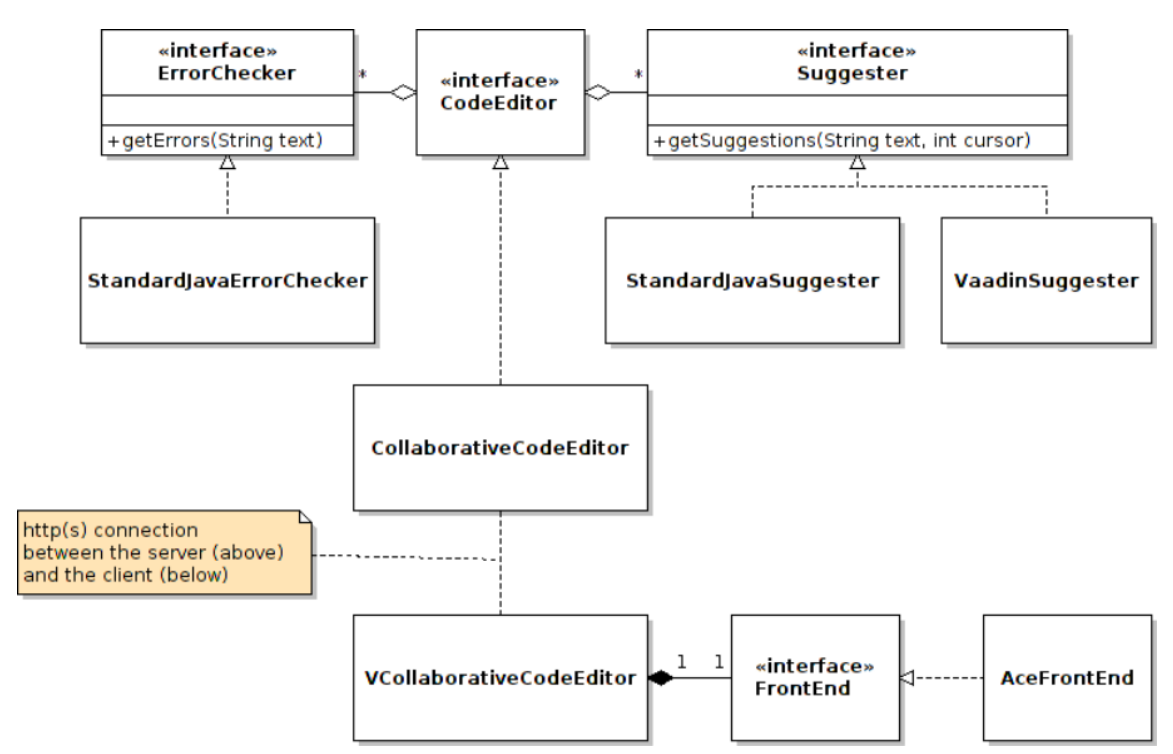

Fig. 3 The General Organization and Structure of the Proposed System

In the process of system implementation, adopt the combination of mode of development. B/S model has the characteristics of "the box", and can be compatible with different operating systems, convenient for teachers and students through online teaching resources, and carry on some simple management operations. $\mathrm{C} / \mathrm{S}$ model has strong ability of transaction processing which can realize the complex business processes, it is mainly used for achieve the function of teachers and administrator user specific requirements, such as teachers courseware and teaching video acquisition, large capacity, or certain types of files FTP upload. In the actual project implementation process, the project schedule change will often happen and need to adjust your plan accordingly. When adjustment, but according to the change of different causes, and take different measures. For external deviation produced by the requirements change, the project manager to take the initiative to excavate the potential needs of customers, avoid to cause changes in the late; The inappropriate for schedule arrangement, can proper adjustment according to the project progress; To the problem of deviation produced by insufficient resources project duration compression method and can be used to solve resource allocation technology.

The original programmer usually begins by setting up a skeleton project ready to accept contributions. Calculation of time between the project tasks tend to be influenced by various factors, such as the relationship between the task, the task of time delay, and project calendar to listen to a day, and so on and so forth. This takes the form of a class or classes with empty definitions or methods without bodies, which the programmer then requests workers to complete. The experience from there is dominated by the strategy used to synchronize code and integrate everyone's contributions. In the following figure 4 , we illustrate the core code blocks of the system.

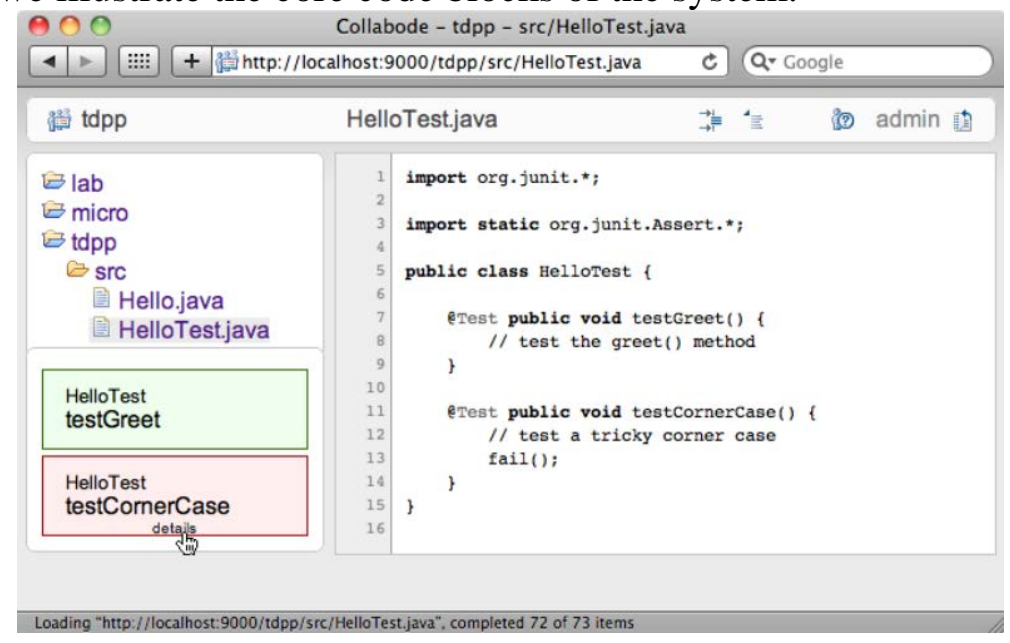

Fig. 4 The Core Code Blocks of the Education System 
The Prospect and In-depth Discussion. System provides the learning based on individual ability and the level of personalized learning environment. Student registration after logging in which can according to the guide of the system to provide the mechanism, browse the course content and online courses, online to submit a job, have a self-test to test their learning effect, to see their knowledge locust weak spot and to adjust their learning strategies, download course related resources and a series of actions. If there is a problem in the process of learning, teachers can participate in, please discuss it in synchronous or asynchronous communication, or in the repository for fuzzy search and if there are no relevant answers in a repository, you can ask the teacher questions by online or leave a message, waiting for the teacher answer questions. Applications are created in the browser-based visual editor that contains both the GUI and the code editor. In the further steps, we will conduct more system optimization work to enhance the current model.

\section{Conclusion and Summary}

In this paper, we design and development of novel English online education and learning system based on B/S architecture. B/S model has the characteristics of "the box", and can be compatible with different operating systems, convenient for teachers and students through online teaching resources, and carry on some simple management operations. Our proposed system combines the machine learning method to enhance the performance. Our paper is a starting point for studying the quality of interface design and the impact of interface design defects on the quality of software application. Our findings implicate researchers and engineers should distinguish classes and interfaces when estimating the quality of software applications. In the future, we will modify the UI of the system.

\section{References}

[1] Keengwe, J., \& Schnellert, G. (2014). Cross-cultural online learning in higher education and corporate training. Register.

[2] Dias S B, Diniz J A. Towards an Enhanced Learning Management System for Blended Learning in Higher Education Incorporating Distinct Learners' Profiles[J]. Educational Technology \& Society.

[3] Jinno, M., Takara, H., Kozicki, B., Tsukishima, Y., Sone, Y., \& Matsuoka, S. (2009). Spectrum-efficient and scalable elastic optical path network: architecture, benefits, and enabling technologies. Communications Magazine, IEEE, 47(11), 66-73.

[4] Tikhonov, D. B., \& Zhorov, B. S. (2012). Architecture and pore block of eukaryotic voltage-gated sodium channels in view of NavAb bacterial sodium channel structure. Molecular pharmacology, 82(1), 97-104.

[5] Guo, C., Lu, G., Li, D., Wu, H., Zhang, X., Shi, Y., ... \& Lu, S. (2009). BCube: a high performance, server-centric network architecture for modular data centers. ACM SIGCOMM Computer Communication Review, 39(4), 63-74. 